\title{
Finding the Optimal Paradigm in Surgical Training and Mentorship: Prof. Saidi's Example
}

Isaac Oluoch Kobe

Surgical Training Fellow- University Hospitals of North Midlands, England

Correspondence to: Isaac Oluoch Kobe P.O Box 58605-00200 Nairobi, Kenya. Email: kobeisaac@yahoo.com

Key words: Mentorship, Journal, Workshops

Ann Afr Surg 2018; 15(2):46 - 47

DOI:http://dx.doi.org/10.4314/aas.v15i2.2

Conflicts of Interest: None
Funding: None

(C) 2018 Author. This work is licensed under the

Creative Commons Attribution 4.0 International License.

I am sure that many of us are still reeling from Prof. Saidi Hassan's demise. All that brilliance, knowledge, humility and dedication gone to waste? Not so, it has been illuminated and passed on to his many mentees. After having heard about Prof. Saidi as a medical intern six years ago, I was pleasantly surprised when I first met him during my surgical training and discovered that instead of a towering physical frame, I found a humble, cheerful and brilliant mind. His great ability to inspire improvement as well as support for weaker younger colleagues is well known. As well, his penchant for seeking to understand social circumstance that affected his trainees' performances and help where he could, was one of his hallmarks as a mentor (1). What has come out to light and surprised many not only within Kenyan medical circles but also beyond, is how he applied this trait and found time for so many people. Furthermore, his ability to provide this personal touch to training and mentorship did not hinder him from achieving numerous accolades, on the contrary, it likely enabled him. Furthermore, he knew how to pull struggling residents out of tense learning environments to get them back on track (1). While the overriding culture in surgical training has been one of intimidation and ridicule, those practising this have been of the erroneously belief that these maladaptive intentions, not based on any evidence, would push surgical trainees to be the best (2). Such has been proven to be counterproductive and is only based on reproducing experiences previously endured by the trainers. On the contrary, mentoring is soon becoming a very important part of the new academic surgical paradigm and the

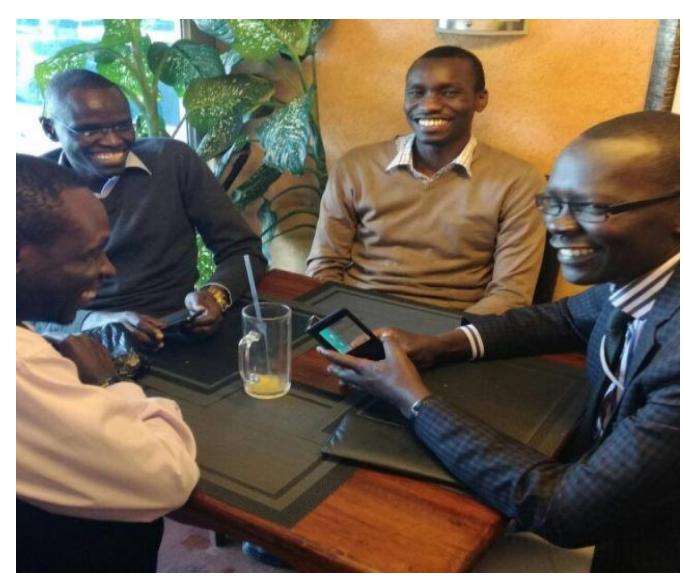

Figure 1: Prof. Saidi with some of his mentees

requirement for mentor based learning as a substitute for rote and experience is increasing (3). It has also been realised that institutions need to provide resources for mentor education in surgical training as studies have proven that it is best delivered in a collegial atmosphere. Over the last decade, the role of mentorship has increased to become a more integral part of surgical education (3).

In reflection, there are several questions that teachers in the surgical profession need to ponder on. What is the best way to inspire learners to put greater effort? What are the best techniques to make the best out of them and ensure efficiency and competencies are up to par with required level of training while at the same time encouraging other important but non -core activities such as research and character building?

Prof. Saidi has done his bit and showed us how he does it in his own way. In the same breath, the new generation of surgical teachers and mentors need to decide which direction we need to take. Likewise, it 
may well be better to appreciate our great mentors in our daily routines rather waiting after the fact, for some calamity, which might not be as insidious as Prof. Saidi's.

\section{References}

1. Bosire N. Guest Newspaper Commentary: Lessons on Mentorship: Daily Nation newspaper 5 Sept 5, 2017; 3

2. Mian A. Review Article: True Mentorship in Medicine: Can Fam 2011; 52(2): 252

3. Platz J. Hyman N. Review article: Mentorship; Clin in Colon Rectal Surg. 2013; 26 (4) 218-23 